\title{
Rectification of Preferences in a Fuzzy Environment
}

\author{
Camilo A. Franco de los Ríos, Javier Montero, and J. Tinguaro Rodríguez \\ Faculty of Mathematics, Complutense University, Madrid 28040, Spain \\ francodelosrios@gmail.com
}

\begin{abstract}
In this paper we consider a complete fuzzy preference structure, defined by means of a constructive approach associated to the necessary learning process in a decision making problem. Preference relations are successively assigned over a given set of alternatives with the possibility of revision and rectification, subject to a certain set of beliefs which sustain the construction of different viewpoints. Measures of information are then considered in this process for a formal identification of uncertainty due to ignorance, a concept that is closely related to ambiguity as defined by Fishburn (1993). Finally, a coherence criterion is proposed in order to study the importance of preferences, in such a way that an indirect and comparative analysis between them is needed so outcomes may be coherently organized.
\end{abstract}

Keywords: fuzzy preferences, rectification, beliefs, viewpoints, uncertainty due to ignorance, ambiguity, coherence measures.

\section{Introduction}

Different notions of preference can be established between distinct classes of entities, actions, circumstances or propositions, in order to understand how decision makers view the world and its inherent uncertainty. Within this vision, motivated by a subjective vision over classical logic [6], the set of possible worlds is viewed as a subset of alternatives, in such a way that preferences are stated on the basis of individual or collective beliefs towards a certain subset of alternatives, taking into account positive and negative arguments for each comparison. In particular, the base of beliefs, a set of formulas where each formula represents an individual belief, delimits the set of possible worlds in such a way that any added belief will impossible worlds be rejected. It is out of question the importance of opening the possibility of revision and rectification of these beliefs, since it is a primary learning characteristic of any intelligent system [18].

In this article the concept of fuzzy environment is supported from a constructive approach providing the setting for fuzzy logic and its applications. In this case, the field of application is preference theory, considering the construction of a preference order, according to a characteristic viewpoint, under uncertainty due to ignorance or ambiguity. The distinction that we follow here is the one that almost a century ago (1921) Knight [15] and Keynes [14] presented between measurable uncertainty (probabilities) and unmeasurable uncertainty (weight of evidence), arguing that ambiguity, 
understood as lack of knowledge [7], [8], has an important role in rational learning and decision processes.

This paper is organized as follows: section 2 discusses the key aspects of uncertainty due to ignorance and reviews ambiguity and coherence as information measures. In section 3, a complete preference structure is defined under characteristic viewpoints. Finally, section 4 examines the possibility of rectification in a decision process with fuzzy preference relations, where information measures have the main function of preserving coherence when knowledge is ambiguous.

\section{Uncertainty and Ignorance}

The search for alternative qualitative and symbolic models is extremely relevant for a further development of preference theory, in order to complement, explain and generalize the classical body of knowledge founded in the theory of (expected) utility. Diverse studies in social sciences (see [7], [10]) where the problem of decision under ignorance is examined according to the approach formulated in [14] and [15], offer some experimental evidence supporting that preferences do not depend only on the degree of uncertainty over the quality of information, but also over its source. This distinction motivates a careful study of uncertainty, stressing the relationship of the concept of ignorance with ambiguity [8] and fuzziness [24], different to probabilistic uncertainty.

To illustrate the idea stated above we recall the classical example of the "two colors" (see [7], [14], [15]). Consider two boxes containing red and black balls. The first box contains 50 red balls and 50 black balls, while the second box has 100 black and red balls without known proportion. Although most decision makers will assign the same probability of reaching for a red ball in any of the boxes $(0.5)$, the weight of the argument in favour of this conclusion is greater in the first case than in the second one. The fact that in general people prefer to bet for the box with known proportion, suggests that subjective probabilities of reaching for a red or black ball are greater in the box where its proportion is known, revealing a behavioural pattern not fully consistent with standard subjective probability and expected utility theory.

As shown in [7], there is a pronounced difference between the type of uncertainty that can be measured by probabilistic functions, which can be referred to as risk, and the type that can not be directly measured by such functions. So, in the presence of uncertainty we have the probability of occurrence of an event, and on the other hand, a degree of confidence over the different attributes of information. Decisions rest upon beliefs about the state of nature in situations where non probabilistic uncertainty is present. In common human decision making, for example, it is clear that probabilistic reasoning gets less weight, since quite often we process mixtures of different kinds of uncertainties (this is the case, for example, of most statistical polls, as soon as they make use of linguistic terms that should bring a specialized non probabilistic analysis).

Restricting ourselves to non probabilistic uncertainty, in particular to a state of knowledge of the decision maker that can be referred to as uncertainty due to ignorance, we realize that such ambiguity affects decisions in a way that contradicts additivity of subjective probability. To clarify this idea we remind below the definition of 
ambiguity [8] and study its relation with fuzziness [24], in order to represent decision situations where the boundary between the set of possible and impossible worlds, constructed upon beliefs that support a finite set of viewpoints, is not always crisp.

Ambiguity, as a primitive concept, has been proposed as a comparative relation on a set of elements $X$ where $P(X)$ is the set of all subsets of $X$. The set measure $\alpha: P(X) \rightarrow[0,1]$ is called an ambiguity function if it satisfies the following three axioms [8]:

$$
\begin{aligned}
& \text { A1. } \alpha(\varnothing)=0 \\
& \text { A2. } \alpha(A)=\alpha\left(A^{\mathrm{c}}\right) \\
& \text { A3. } \alpha(A \cup B)+\alpha(A \cap B) \leq \alpha(A)+\alpha(B)
\end{aligned}
$$

Minimum ambiguity is assigned to the null set, such that $\alpha(\varnothing)=\alpha(X)=0$. The complement of $A$ is denoted by $A^{\mathrm{c}}$, so the central idea of this characterization is expressed by (A2), where ambiguity is a measure of a certain attribute of information shared by any set $A$ and its complementation. Let us remind that for probabilities, if $p$ stands for a probability function, $p(A)+p\left(A^{\mathrm{c}}\right)=1$, while for ambiguity, $\alpha(A)=\alpha\left(A^{\mathrm{c}}\right)$, reflecting the original intuition [8] that whatever underlies the ambiguity of a set also underlies the ambiguity of its complement. Finally, the third axiom expresses submodularity: the idea that the union of two sets $A, B$ may reduce or cancel ambiguities associated to each one (considered separately), in such a way that if $A$ and $B$ are disjoint, then $\alpha(A \cup B) \leq \alpha(A)+\alpha(B)$ and if $\alpha(A \cup B)=X$ then $\alpha(A \cap B) \leq \alpha(A)+\alpha(B)$.

The above idea of ambiguity measures can be translated into fuzzy set theory [25]. Fuzzy logic focuses on situations where the boundary between the set of possible and impossible worlds is not always crisp, and a fuzzy subset $R$ may be naturally described by the membership and non-membership degree of each element $x$ in $X$, denoted by $R^{+}(x)$ and $R^{-}(x)$ respectively. As suggested in [24], fuzziness may rise from the lack of an absolute distinction between $R^{+}$and $R^{-}$. Note that $R^{-}$does not necessarily correspond to the classical conception of complementation, since (see for example [2], [16], [19]) non-membership intensities can be better understood as some kind of orthogonal but positively measurable degrees over the elements of $X$. A more detailed study on fuzziness and its different interpretations for fuzzy sets ([25], [26]) and intuitionistic fuzzy sets [2] can be found in [22].

Following [24], where $R^{-}$is taken as the complement in such a way that $R$ $(x)=R^{\mathrm{c}}(x)=1-R(x)$, if we assume that $R$ is a fuzzy subset defined over the finite set $X$ with cardinality $n$, the intersection of $R^{+}(x)$ and $R^{-}(x)$ is defined by the set $I(x)$, $I=R^{+} \cap R^{-}$(see [4] in order to stress the relevance of the related concept of overlapping when dealing with fuzzy information). As a result, if the set $I(x)$ is empty, then $R^{+}$and $R^{-}$are clearly distinct and no fuzziness occurs (membership intensities are either 0 or 1 ) but when $I(x)$ is positive, the larger it becomes, the greater the fuzziness associated with the set $R$.

Let us remind the formal characterization of measures of fuzziness, $\operatorname{Fuzz}(R)$, for any fuzzy subset $R$ (see [24]):

$\mathrm{F} 1 . \operatorname{Fuzz}(R)=0$ if and only if $R$ is a crisp subset.

$\mathrm{F} 2$. $\operatorname{Fuzz}(R)$ has its maximal defined as $R(x)=0.5$ for all $x$. 
F3. For any fuzzy subset $R^{*}$ such that $R^{*}(x) \geq R(x)$ if $R(x) \geq 0.5$ and $R^{*}(x) \leq R(x)$ if $R(x) \leq 0.5$ then $\operatorname{Fuzz}(R) \geq \operatorname{Fuzz}\left(R^{*}\right): R^{*}$ is called a sharpened version of $R$.

As shown in [24], a measure of fuzziness can be also an ambiguity measure in the sense of Fishburn [8], where $R(x) \cup S(x)=\max (R(x), S(x)), R(x) \cap S(x)=\min (R(x), S(x))$ and $R^{\mathrm{c}}(x)=1-R(x)$ for any fuzzy sets $R$ and $S$. As a consequence, ambiguity measures are also valid for measuring fuzziness.

Once the concept of uncertainty due to ignorance has been associated to the concept of ambiguity and fuzziness, we can analyze its role in a preference ordering construction process. Usually preference evaluation vía binary relations considers only the two alternatives at stake and their principal attributes, paying less attention to second order characteristics, which receive greater relevance in situations where ambiguity is present. For this reason we need to analyze the different states of knowledge (from absolute certainty to total ignorance) of a decision maker.

A comparative analysis should allow us to distinguish between different states of knowledge, judging the set of constructed preferences based on expert knowledge or maximal certainty. In consequence, diverse attributes may receive positive and higher values of relevance in a comparative evaluation than in an isolated one. In order to study the importance of preference relations and their outcomes, it is necessary to remind some basic definitions and introduce a preference structure within a fuzzy environment.

\section{Complete Preference Structure}

A decision making problem is here understood as the construction of an ordering process over a finite set of alternatives $A$, where fuzzy binary relations are defined between alternatives $a$ and $b$ by a degree of truth for the predicate $R$, " $a$ is at least as good as $b$ ", assigned according to a complete and partially ordered valuation set $L$. Following standard approaches (see [9], [17]), each fuzzy preference relation $R$ can be understood as a composition of four different relations and its corresponding intensities. These components are "strict preference of $a$ over $b$ ": $P$, "strict preference of $b$ over $a$ ": $P^{-1}$, "indifference between $a$ and $b$ ": $I$, and "incomparability between $a$ and $b ": J$.

A fuzzy preference binary relation for a set of alternatives $A$ is characterized by a complete valuation space $L$ and a function $R$ such that $R: A \times A \rightarrow L$. For simplicity, we consider the case in which $L=[0,1]$. Here we consider the three axioms proposed in [9] and [17]. The "Independence of Irrelevant Alternatives" axiom states that for every pair of alternatives $a, b$, the values of $P(a, b), I(a, b)$ and $J(a, b)$ depend only on the values of $x=R(a, b)$ and $y=R(b, a)$. The existence of the continuous functions,

$$
p, i, j:[0,1]^{2} \rightarrow[0,1]
$$

can be stated, in such a way that $P(a, b)=p(x, y), I(a, b)=i(x, y)$ and $J(a, b)=j(x, y)$. The "Positive Association" axiom says that functions $p(x, n(y)), i(x, y)$ and $j(n(x), n(y))$ are non-decreasing over both arguments. The "Symmetry" axiom states the symmetry of the functions $i(x, y)$ and $j(x, y)$. The complete preference structure is therefore described as: 


$$
R(x, y)=\langle p(x, y), p(y, x), i(x, y), j(x, y)\rangle
$$

where $R(x, y) \geq 1$. In this way, the aggregated intensity is being decomposed into four intensities that should cover the total possible intensity.

In order to build up the relation $R$ over the available alternatives, a rational process is needed to identify viewpoint $h \in H$, under which preferences can be valued. The principle of rationality that we follow here is the one of minimum action, where an individual searches for the viewpoint with lowest dimension (minimal number of criteria) so a consistent preference ordering can be constructed for a finite subset of alternatives. For an exploration of different approaches to dimension for some classes of orders see for example [12].

Each viewpoint $h$ is constructed over the attributes of the available information on the elements of the set $A$, interpreted according to the base of beliefs $B$, over which the set of criteria $C$ can be defined. Here $h$ represents an independent state of mind for undertaking the preference analysis. The following definition is based on [13]:

Definition. A viewpoint $h \in H$ for a subset of alternatives $S_{h} \subseteq A$ is characterized by an outcome space $\Omega_{h}$, a set of criteria $C_{h}$ where each criterion $c_{h}: S_{h} \rightarrow \Omega_{h}$ maps alternatives to their outcomes, and a partial order given by the set of fuzzy preference relations $R$ over $\Omega_{h}$.

The set of criteria $C_{h}$ is a finite set whose elements can be combined for the construction of any viewpoint $h \in H$, where each criterion determines a new dimension for the outcome space $\Omega_{h}$.

Differences between viewpoints $h, v \in H$ over the same set of alternatives $S$, case where $S_{h}=S_{v}$, may refer to differences in preferences, in outcome spaces or in criteria [3], [13]. If a set of alternatives $S$ is organized by a unique viewpoint $h$, we say it is the characterizing viewpoint of $S$, denoted by $S_{h}$. If more viewpoints are candidates for ordering the same set $S$, then we say that a process of rectification is possible. Rectification allows the identification of a new order on the alternatives in $S$, revealing a change in the preferences of the decision maker. When an intelligent agent rectifies, a new viewpoint is being searched. This is a task where the decomposition or combination of viewpoints may be necessary. Therefore, two viewpoints can be combined or decomposed for constructing a new enlarged or reduced viewpoint, where the set of alternatives, preferences, outcome spaces or criteria may change. Still, maximum coherence needs to be preserved.

The complete structure for this preference constructive approach can now be defined as,

$$
Z=\langle A, H, R, L\rangle
$$

where $H$ is the set of all possible viewpoints under which the alternatives in $A$ can be completely ordered according to the structure given by $R$ and its valuation set $L$. 


\section{Information Measures and Preference Rectification}

The process of decision making implies a comprehensive identification of the most relevant attributes of information, learning how to order the available alternatives and classifying them (see [1], [16]) with a maximum degree of coherence. Stressing the importance of a comparative analysis, a decision making process is here called a learning process if and only if rectification of preferences is possible. Rectification is then understood as the reconstruction of a preferential order for a finite set of alternatives, where it may be necessary an expansion (composition), or on the contrary, a contraction (decomposition) of viewpoints.

When any pair of alternatives is compared and a preference relation is constructed, an order is assigned over $S \subseteq A$ according to certain viewpoint $h$. Following the idea that a viewpoint may have multiple extensions so a preferred outcome may at last be found [13], the decision maker has to be able to rectify along the sequential learning process. Such learning process is here characterized as the elicitation of knowledge from single attributes of information, where these attributes are identified step by step. In this way, the most relevant attributes can change and viewpoints may need modification (combined, contracted or enlarged).

The sequential learning process described above needs to take into account its natural ambiguity or degree of ignorance. Let us take for example two viewpoints $h$ and $v$. If we compare two alternatives $a, b$ under both viewpoints, and the intensity of the predicate " $a$ is at least as good as $b$ under viewpoint $h$ ", $R_{h}(a, b)$, is stronger than the intensity of $R_{v}(a, b)$, then we are less certain about the value of $R(a, b)$ when the viewpoint $v$ is used than when $h$ is used. This means that $v$ is more ambiguous than $h$.

Following [26], [27], we can measure if there is a possibility that $R_{v}(a, b)$ is true given that we know $R_{h}(a, b)$. This possibility measure (see [6], [27]),

$$
\rho_{R_{h}(a, b)}\left(R_{v}(a, b)\right)=\max \left(R_{w}(a, b)\right)
$$

expresses the degree of intersection between $h$ and $v$, where $w=h \wedge v$. Analogously, we can measure if there is a possibility that $R_{v}^{-}(a, b)$, where $R^{-}(x)=R^{\mathrm{c}}(x)=1$ $R(x)$, is true given that we know $R_{h}(a, b)$. The measure of certainty [27] (also called measure of necessity [6])

$$
\varsigma_{R_{h}(a, b)}\left(R_{v}(a, b)\right)=1-\rho_{R_{h}(a, b)}\left(R_{v}^{-}(a, b)\right)
$$

expresses the degree of inclusion of $h$ in $v$. In addition,

$$
v_{R_{h}(a, b)}\left(R_{v}(a, b)\right)=\rho_{R_{h}(a, b)}\left(R_{v}(a, b)\right)-\zeta_{R_{h}(a, b)}\left(R_{v}(a, b)\right)
$$

is an ambiguity measure as defined in A1-A3 (see [24]), where the greater that $v$ is, the more ambiguity or uncertainty due to ignorance exists. In this way, when ambiguity on viewpoint $h$ is large, a greater possibility exists for rectifying over the current order given by $R_{h}(a, b)$. This measure helps to identify when some preference order rests upon a viewpoint with a low degree of confidence. A new viewpoint that takes 
into consideration other attributes, second order characteristics, can be necessary for a robust decision process to take place.

Now, if we want to assign a greater value of confidence to the viewpoint with a greater degree of truth, we need a measure of confidence, dual of uncertainty (due to ignorance), such that a greater value can be assigned to the outcome that uses information with a higher intensity of certainty. The idea is to find a measure that explicitly assigns, by a comparative analysis, a greater value if a high level of confidence exists. Such a measure, closely related to ambiguity as it has been examined above, will directly distinguish the agent's states of knowledge, by a confidence scale that goes from total ignorance to total certainty, based on expert knowledge or maximal certainty.

We then propose that the confidence measure we are looking for can be analyzed as a coherence measure. Recall that ambiguity [8] and coherence measures [20] are explicitly related by extension theorems proven for any strong negation (such that a kbijection verifying $n(x)=k-1(k(1)-k(x))$ exists, see [5], [20], [21]). These coherence measures evaluate how fuzzy and similar are any pair of fuzzy sets, where the degree of fuzziness is characterized by F1-F3. For our purposes, the basic idea can be phrased as the nearer a fuzzy preference relation is to the sets $\varnothing$ or $\Omega$, the higher coherence measure is expected.

Given the referential set $X$, with $R_{j}, R_{i} \in X$, representing the set of all fuzzy preference relations $Q^{f}(X)$ and given a strong negation $n$ on $Q^{f}(X)$, coherence measures can be then defined over the set of fuzzy preferences: the function $\zeta: Q^{f}(X) \times Q^{f}(X) \rightarrow[0,1]$ is a coherence measure, if and only if the following three axioms are satisfied (see [20], [21]):

C1. $\zeta\left(R_{i}, R_{j}\right)=\zeta\left(R_{j}, R_{i}\right)$

C2. $\zeta\left(R_{i}, n\left(R_{j}\right)\right)=n\left(\zeta\left(R_{j}, R_{i}\right)\right)$

C3. $\zeta(\varnothing, X)=0$

The first condition (C1) states the symmetry of this measure and the third one (C3) guarantees minimum coherence. About (C2), analyzing the relation between ambiguity and coherence measures, we can see a complementary approach between A2 and C2 2 in the sense that the coherence between $R_{i}$ and $R_{j}^{-}$is the opposite of the existing one between $R_{i}$ and $R_{j}$. Remind that if we want to assign a greater value of confidence to the viewpoint with a greater degree of truth, we need some measure of confidence, something like the dual of ambiguity. Therefore, coherence is here understood as a comparative confidence measure for any pair of fuzzy sets, in our case preference relations, such that a common link between fuzzy preference relations can be identified for any viewpoint.

Let us recall the above ambiguity measure $v_{x}(y)$, taking $x=R_{h}(a, b)$ and $y=R_{v}(a, b)$. If ambiguity is represented by the difference between possibility and 
necessity measures, then confidence should take into account the aggregated weight of these two measures. And examining confidence as here presented, we can pursue a coherence measure close to

$$
\frac{1-v_{x}(y)}{2}
$$

Therefore, if $\quad v_{x}(y)=\rho_{x}(y)-\varsigma_{x}(y) \quad$ and $\quad \rho_{x}(y)=\max (x, y) \quad$ and $\varsigma_{x}(y)=1-\max \left(x, y^{c}\right)$, then $v_{x}(y)=\max (x, y)+\max \left(x, y^{c}\right)-1$. Condition $\mathrm{C} 1$ requires that the measure is symmetric, but it can be seen that for the necessity measure the order of the arguments does matter. In other words, the degree of inclusion of $x$ in $y$ is not the same as the degree of inclusion of $y$ in $x$ : $1-\max \left(x, y^{c}\right) \neq 1-\max \left(y, x^{c}\right)$. For this reason, because coherence is conceived as a relation defined over pairs of fuzzy sets, the max function can be replaced by some kind of distance representing how far is each set from any other one.

If we take for example the Euclidian distance $d(x, y)=\frac{1}{m} \cdot \sum_{i=1}^{m}\left|x_{i}-y_{i}\right|$, the measure defined by (2), where $d(x, y)$ and $d\left(x, y^{c}\right)$ represent a separation degree between $x$ and $y$ and between $x$ and everything not being $y$, respectively, becomes

$$
\beta(x, y)=\frac{1+d\left(x, y^{c}\right)-d(x, y)}{2} .
$$

Coherence of fuzzy preferences, as defined by C1-C3 and (3), has been initially explored in [11] (the origin of (3) as a coherence measure is founded in [20]), establishing a possible criterion where all outcomes can be evaluated. This proposal is useful for any viewpoint, offering relevant information about the importance of its preferences, according to the confidence on their truth values. In order to maintain coherence along the learning process, this coherence criterion allows the decision agent to add an extra dimension to the outcome space of a given viewpoint (see example 1 below), and it can also be used to examine the degree of coherence between different viewpoints so the possibility of combining, contracting or enlarging them can be studied (see example 2 below).

Example 1. Under the state of knowledge of uncertainty because of ignorance, the coherence criterion enables the decision agent to assign an ordinal value over the set of alternatives, judging the set of constructed preferences against one certain relation $R^{*}$, which stands as an organizing predicate [23] and denotes maximal certainty or expert knowledge. Given the existence of such a predicate, there exists an associated coherence criterion for any viewpoint, establishing an independent dimension where the outcome space can be coherently organized. 
Consider the set of preference relations $R_{h}$ under viewpoint $h$ such that a decision agent is interested in identifying weak preference with a greater degree of confidence (case where it is difficult to differentiate strict preference from indifference). Therefore, for any preference relation where the components $p$ and $i$ have simultaneously high membership intensities, a higher degree of coherence, as defined by $\mathrm{C} 1-\mathrm{C} 3$, will be assigned to it. In order to apply the coherence criterion we use an organizing predicate $R^{*}$ such as $R^{*}=\{1,0,1,0\}$. As it can be seen, $R^{*}$ represents the most certain knowledge for the values defined by (1).

Now, consider the following fuzzy preference relations:

$$
\begin{aligned}
& R_{h}(x, y)=\{0.5,0.5,0.5,0.0\} \\
& R_{h}(w, z)=\{0.9,0.8,0.8,0.1\} \\
& R_{h}(u, z)=\{0.2,0.2,0.9,0.7\}
\end{aligned}
$$

This set of preferences reveals some uncertainty due to ignorance and notice that there is not a complete order between them. Comparison of preference is missing between alternatives $(x, z)$. Through a third element it would be possible now to examine how these alternatives relate with each other. According to the coherence criterion (3), the corresponding confidence-ordinal values over these set of fuzzy preferences relations under viewpoint $h$ are:

$$
\begin{gathered}
\beta\left(R_{h}(x, y), R^{*}\right)=0.625 \\
\beta\left(R_{h}(w, z), R^{*}\right)=0.70 \\
\beta\left(R_{h}(u, z), R^{*}\right)=0.55
\end{gathered}
$$

In this case, the optimal outcome is $R_{h}(w, z)$, because it has a greater coherence measure than all the others. The coherence criterion, which is here presented as a dimension where preferences may be organized according to the degree of confidence on their truth values, identifies the fuzzy preference relation with the strongest intensity and certainty.

Example 2. Let's consider three viewpoints $h, v, w \in H$ over the same set of alternatives $a, b \in A$, such that both $a, b$ are members of $S_{h}, S_{v}$ and $S_{w}$. Remember that we say that a process of rectification is possible when two or more viewpoints are candidates for ordering the same set of alternatives. In this case $S_{h}=S_{v}=S_{w}$, so if an intelligent agent rectifies then his viewpoint changes. For this reason some decision aid is necessary for the agent to identify the optimal viewpoint to be used, based in his beliefs but also on the confidence (over his state of knowledge) that the set of preferences reveal. The viewpoint may change, but maximum coherence needs to be preserved.

Assume the agent believes viewpoint $h$ is the most important, but when evaluating preference between $a$ and $b$ under $h$, confidence in its outcome suffers of some ambiguity. This can be caused by many reasons, for example, if $h$ is composed by price-quantity criteria, the agent may suffer from lack of perfect knowledge over price stability. So, if the agent is comparing different goods, he can also reveal his preferences under viewpoint $v$, composed by fair-trade attributes, and viewpoint $w$, which values presentation 
and marketing. The agent will then have to decide and the viewpoint he uses will determine the final outcome, which has to be an optimal answer to his necessities.

Consider the following preferences,

$$
\begin{aligned}
& R_{h}(a, b)=\{0.7,0.3,0.5,0.3\} \\
& R_{v}(a, b)=\{0.2,0.8,0.2,0.4\} \\
& R_{w}(a, b)=\{0.9,0.2,0.9,0.1\}
\end{aligned}
$$

If the agent wants to choose the most certain viewpoint, such that alternative $a$ is at least as good as alternative $b$ with maximum certainty, he can use the same organizing predicate $R^{*}$ defined in example 1 . Otherwise, a confidence order can be constructed between viewpoints $h, v$ and $w$ so the possibility of merging viewpoints, due to their relative coherence, can be examined. Using the coherence measure defined in (3):

$$
\begin{array}{cc}
\beta\left(R_{h}, R^{*}\right)=0.65 & \beta(h, v)=0.425 \\
\beta\left(R_{v}, R^{*}\right)=0.30 & \beta(h, w)=0.65 \\
\beta\left(R_{w}, R^{*}\right)=0.875 & \beta(v, w)=0.30
\end{array}
$$

As a result, the optimal outcome according to $R^{*}$ is given by viewpoint $w$ and the agent will find reasons to rectify. In the other case and complementary to the situation just described, the decision maker will be also motivated to combine viewpoints $h$ and $w$, in order to find a new enlarged viewpoint where the decision maker can be more certain about his/her knowledge and preferences.

\section{Final Comments}

A complete fuzzy preference structure has been considered for decision making, characterized as a learning process, where preference relations need to be successively assigned over a set of alternatives, always allowing revision and rectification of beliefs. An analytical framework has been set for studying preference construction and the identification of an optimal outcome under characterizing viewpoints.

\section{References}

1. Amo, A., Montero, J., Biging, G., Cutello, V.: Fuzzy classification systems. European Journal of Operational Research 156, 459-507 (2004)

2. Atanassov, K.: Intuitionistic Fuzzy Sets. Theory and Applications. Physica-Verlag, New York (1999)

3. Bouyssou, D., Marchant, T., Pirlot, M., Tsoukiàs, A., Vincke, P.: Evaluation and Decision Models with Multiple Criteria, vol. 86. Springer, Heidelberg (2006)

4. Bustince, H., Fernández, J., Mesiar, R., Montero, J.: Overlap function. Non Linear Analysis $72,1488-1499$ (2010)

5. Cubillo, S., Bellido, S.: A first approximation to Ng-coherence measures. In: Proceedings EUSFLAT 2003, pp. 709-713 (2003) 
6. Dubois, D., Prade, H.: Formal representations of uncertainty. In: Bouyssou, D., Dubois, D., Pirlot, M., Prade, H. (eds.) Decision-Making Process. ISTE, London (2009)

7. Ellsberg, D.: Risk ambiguity and the savage axioms. Quarterly Journal of Economics 75, 643-669 (1961)

8. Fishburn, P.: The axioms and algebra of ambiguity. Theory and Decision 34, 119-137 (1993)

9. Fodor, J., Roubens, M.: Fuzzy Preference Modelling and Multicriteria Decision Support. Kluwer Academic Publishers, Dordrecht (1994)

10. Fox, C., Tversky, A.: Ambiguity aversion and comparative ignorance. The Quarterly Journal of Economics 110, 585-603 (1995)

11. Franco de los Ríos, C., Montero, J.: On the use of coherence measures for fuzzy preference relations. In: Eurofuse Workshop 2009, pp. 15-19 (2009)

12. González-Pachón, J., Gómez, D., Montero, J., Yáñez, J.: Soft dimension theory. Fuzzy Sets and Systems 137, 137-149 (2003)

13. Junker, U.: Preferences in an open world. In: First International Conference, ADT 2009, pp. 215-224 (2009)

14. Keynes, J.: A Treatise on Probability. MacMillan, London (1963)

15. Knight, F.: Risk, Uncertainty, and Profit. University of Chicago Press, Chicago (1971)

16. Montero, J., Gómez, D., Bustince, H.: On the relevance of some families of fuzzy sets. Fuzzy Sets and Systems 158, 2429-2442 (2007)

17. Montero, J., Tejada, J., Cutello, C.: A general model for deriving preference structures from data. European Journal of Operational Research 98, 98-110 (1997)

18. Nebel, B.: A knowledge level analysis of belief revision. In: Proceedings of the 1st International Conference on Principles of Knowledge Representation and Reasoning, pp. 301311 (1989)

19. Öztürk, M., Tsoukiàs, A.: Modelling uncertain positive and negative reasons in decision aiding. Decision Support Systems 43, 1512-1526 (2007)

20. Sancho-Royo, A., Verdegay, J.: Coherence measures on finite fuzzy sets. International Journal of Uncertainty, Fuzziness and Knowledge-based Systems 8, 641-663 (2000)

21. Sancho-Royo, A., Verdegay, J.: Fuzzy coherence measures. International Journal of Intelligent Systems 20, 1-11 (2005)

22. Szmidt, E., Kacprzyk, J.: Entropy for intuitionistic fuzzy sets. Fuzzy Sets and Systems 118, 467-477 (2001)

23. Trillas, E.: On the use of words and fuzzy sets. Information Sciences 176, 1463-1487 (2006)

24. Yager, R.: On a measure of ambiguity. International Journal of Intelligent Systems 10, 1001-1019 (1995)

25. Zadeh, L.: Fuzzy sets. Information and Control 8, 338-353 (1965)

26. Zadeh, L.: The concept of a linguistic variable and its application to aproximate reasoning. Information Sciences 8, 199-249 (1975)

27. Zadeh, L.: Fuzzy sets and information granularity. In: Adavances in Fuzzy Set Theory and Applications, pp. 3-18. North-Holland, Amsterdam (1979) 